\title{
Statisticians roll up your sleeves! There's a crisis to be solved.
}

\author{
Statisticians play a key role in almost all scientific research. But are they also the key to \\ solving the reproducibility crisis? Heidi Seibold, Alethea Charlton, Anne-Laure Boulesteix \\ and Sabine Hoffmann urge statisticians to take an active role in promoting more replicable \\ and more credible science.
}

Imagine waking up in 2030, after a 10-year hibernation, to discover that the scientific community has completely changed. Instead of running multiple analyses to selectively report those that yield significant results ("fishing for significance" or "p-hacking") and presenting post-hoc hypotheses as a priori ("HARKing"), researchers respect the steps they specified in preregistered analysis plans. They also commonly submit registered reports in which preregistered proposals are evaluated prior to data collection. As a consequence, journal editors and reviewers can evaluate their work based on its originality and methodological rigor, rather than based on the statistical significance of their results. Publication bias is a thing of the past. Moreover, researchers routinely assess and report the robustness of their findings, publish (both failed and successful) replication studies and make their code and data available allowing others to reuse and reproduce their work. Overall, the research process is efficient, transparent and credible, both within the scientific community and beyond.

This picture contrasts starkly with the current state of the scientific community. We are experiencing what is referred to as the replication crisis, reproducibility crisis or simply statistical crisis in science. Researchers are incentivized to use questionable research practices (including "The seven sins of significance tests" by Held and Schwab 2020) and replication attempts across many disciplines have shown that research findings are far less reliable than we previously assumed. The common misuse and misinterpretation of $p$-values has led to numerous admonishing statements issued by the American Statistical Association and others (Colquhoun 2014; Greenland et al. 2016; Wasserstein et al. 2016; Wasserstein et al. 2019). Some journals have even abolished inferential statistics (Trafimow and Marks 2015). In preclinical research alone, approximately 28 billion dollars are spent annually on research findings that are not replicable (Freedman et al. (2015)). Public opinion on important societal questions concerning, for instance, climate change or the current COVID-19 pandemic is largely influenced by social media, rather than reflecting the scientific consensus on these topics. While there is an urgent need for the scientific community to become more efficient, replicable and credible, it seems that it would take nothing less than a miracle for this change to happen during your 10-year hibernation.

In our recent article in Significance (Boulesteix et al. (2020)), we addressed what we refer to as a "replication crisis in methodological research" and presented ideas on what statisticians 
can learn from other disciplines in solving this crisis. Here, we examine another perspective, focussing on the skills and good practices that statisticians can bring to the table for the research community as a whole. We argue that instead of falling asleep and waiting for others to do the job or merely finger-pointing at bad practices in other fields, we as statisticians have a tremendous role to play in solving the statistical crisis in science. To illustrate the concrete steps we can take to contribute to the revolution that would make research more efficient, transparent and credible, we present you with role model statisticians: The applied statisticians (see Figure 1) and the methodological statisticians (see Figure 2) of tomorrow.

\section{The applied statisticians of tomorrow}

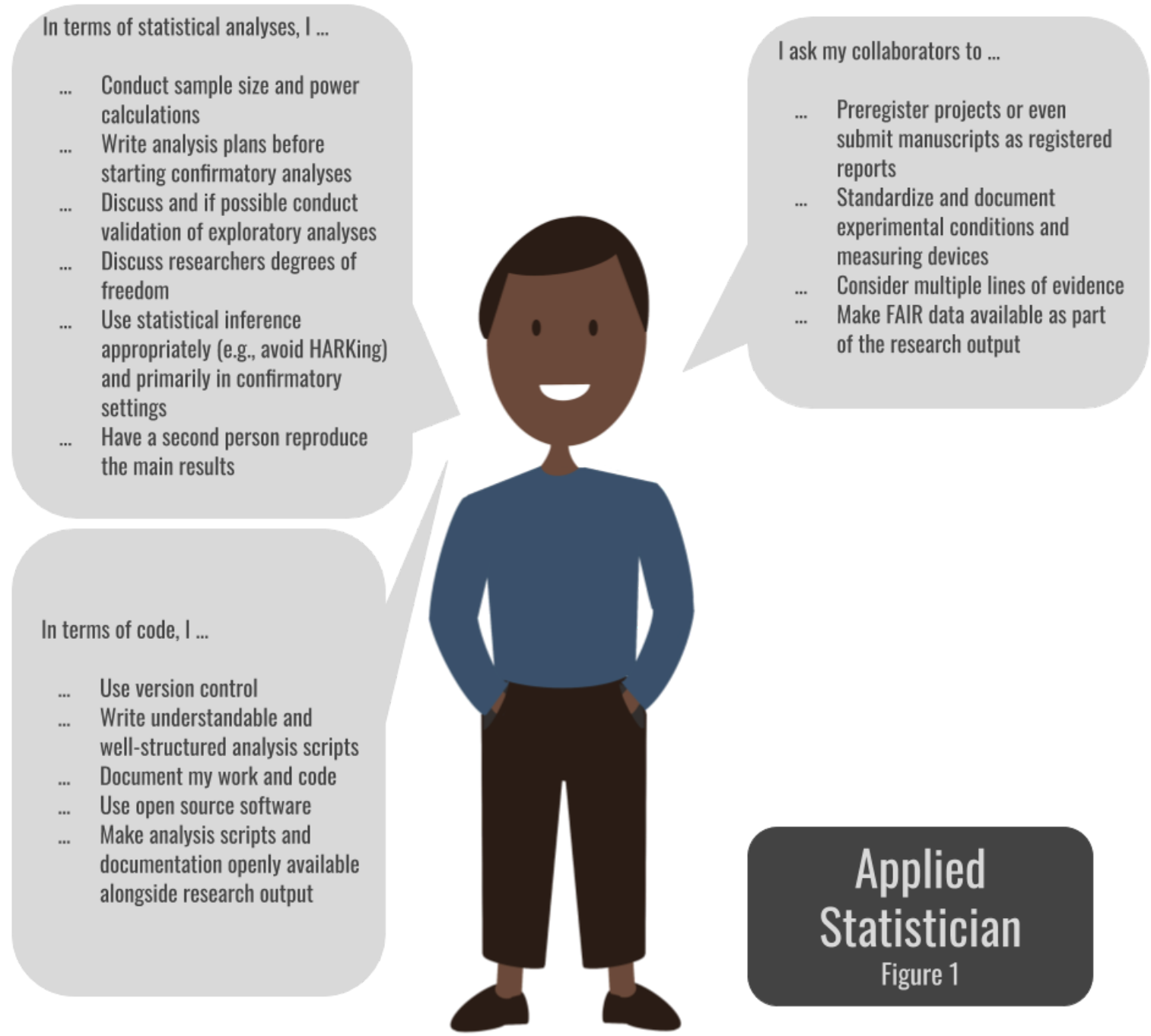

Applied statisticians are responsible for statistical planning and statistical analysis, within research projects or as statistical consultants. They play a major role in research and 
reporting processes and the quality of their work strongly impacts computational reproducibility, replicability and the robustness of research findings.

The applied statisticians of tomorrow routinely perform power calculations and write statistical analysis plans for confirmatory research, ideally as part of preregistration, in order to improve the statistical power of the studies and avoid p-hacking and HARKing (Figure 1, upper left). In exploratory settings, where it is impossible to fully specify all analysis steps, they interpret the results with great caution and adopt validation strategies using independent data to make the results more credible (Daumer et al. 2008).

The applied statisticians of tomorrow are aware that researchers will come up with a wide variety of analysis strategies, even when they are asked to answer the same research question on the same data set (as was recently done by Silberzahn et al. 2018, and Botvinik-Nezer et al. 2020). These strategies will yield different results. To assess the robustness of research findings to these "researcher degrees of freedom" (see Simmons et al. 2011) - which may include model, method and data pre-processing choices - applied statisticians can use classical sensitivity analyses but also recently developed frameworks which allow systematic and comprehensive reporting of the results of alternative analysis strategies (Hoffmann et al. 2020).

Have you ever tried to rerun an analysis code you wrote 6 months ago and found that you are unable to reproduce your results? To minimize these frustrating experiences (and be more efficient), the applied statisticians of tomorrow write high quality code, use version control and document their code well (Figure 1, lower left). To make work more transparent and credible and to allow others to rerun and reuse analysis scripts, software and scripts are published as open source (choices which are increasingly encouraged or even required by funding agencies, e.g. Horizon Europe). Finally, the applied statisticians of tomorrow verify that a research compendium (i.e. a scientific publication including data, code, and documentation, see Nüst et al. 2017) is reproducible by giving it to an independent researcher. They rerun all scripts and obtain the same results without talking to anyone who was involved in the initial analysis.

We can improve our own work, but as statisticians we also collaborate with other researchers and can have a positive impact on them (Figure 1, right-hand side). The important role of statisticians in applied research projects gives the applied statisticians of tomorrow leverage to require good scientific practices from collaboration partners, even if these practices are only indirectly related to statistics (see, e.g Manamley et al. 2016). By encouraging collaborators to consider multiple lines of evidence and to standardize and document experimental conditions and measuring devices, the probability of obtaining a surprising finding simply by chance is minimized, and it is more likely that others will be able to reproduce the experiment. Preregistration of the research project, or even a submission as a registered report, can minimize the risk of p-hacking, HARKing and publication bias. By publishing data as part of the research output, the applied statisticians of tomorrow and their collaboration partners give others the possibility of reusing data and verifying results. This 
makes work more transparent and credible, but also speeds up the process of research by avoiding unnecessary duplication of time-intensive tasks. Open data is useless when it is only available from a hidden website or in a proprietary format, therefore the applied statisticians of tomorrow make data, not only open, but also FAIR (findable, accessible, interoperable and reusable, see https://www.go-fair.org/fair-principles). Even in cases where raw data cannot be made openly available, e.g., due to privacy concerns, it is still useful to FAIRify the data as much as possible (Jacobsen et al. 2020). For example, by providing the metadata on a data platform (potentially with the option to apply for data access).

\section{The methodological statisticians of tomorrow}

Methodological statisticians are responsible for developing and investigating statistical analysis methods. They decide which new methods to develop, how to implement them and make them available to potential users. In an ideal world, they are supported by software experts who understand the field (i.e. research software engineers or RSEs for short). From this short description, it's clear that both methodological statisticians and RSEs have a major role to play in improving reproducibility and replicability in science.

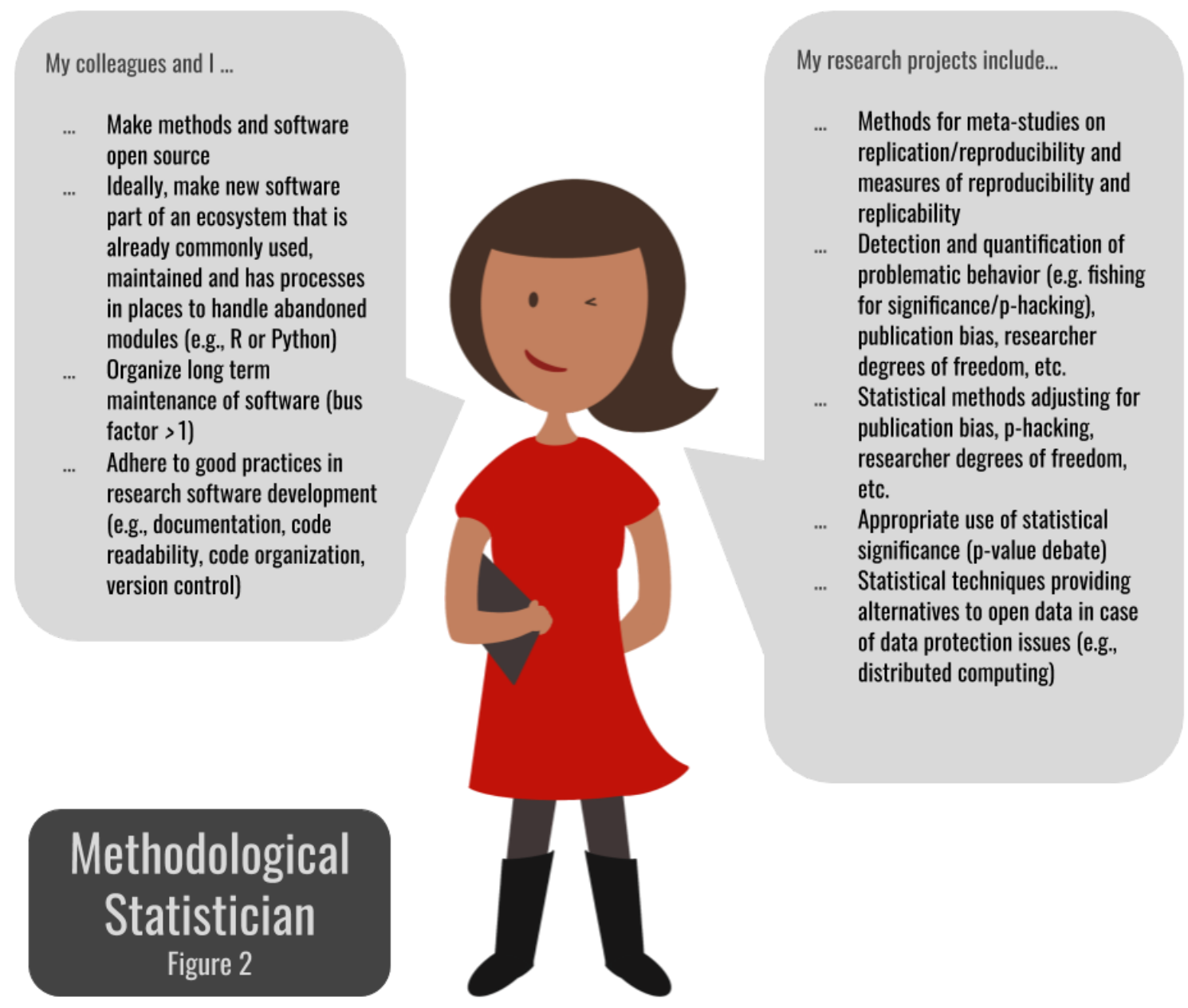


The methodological statisticians of tomorrow make the methods and software they develop available in a form that supports reproducibility (see Figure 2, left-hand side). Software should be open source and sustainable. If, 5 years later, statistical software is no longer available or becomes unusable because it is not maintained, it will be impossible for anyone to reproduce the results. In the same vein, if the background code changes but there is no change log or versioning of the software, it will be impossible to pinpoint exactly why subsequent results differ from the original study. It is therefore essential that research software sticks to good software practices (Hastings et al. 2014): Use of version control, good documentation and compliance with coding standards.

If you've ever stopped to think about reproducibility and replicability you've probably wondered how we can measure these concepts. Is a result reproducible only if exactly the same number is obtained? Or is a small difference acceptable? If so, how much? Statisticians can play an important role in finding answers to these questions. The methodological statisticians of tomorrow play an active role in developing new methods to answer the most burning questions in research replicability, reproducibility and open science. Figure 2, right-hand side, provides examples of relevant topics in this area. These are essentially methodological research topics with a metascientific dimension. Additionally, statisticians can also contribute to other metascientific projects, such as surveys on current (statistical) practice.

Developing statistical methods to detect both problematic scientific behavior, as well as phenomena such as publication bias or researcher degrees of freedom, are an important contribution to the scientific community. For example, measuring publication bias is a first step in later adjusting for it, a process that increases the credibility of the whole field of meta-analyses. This is - to an extent - already being done, but current methods show a number of shortcomings and much work is left to do (see, e.g. Nelson 2018). Recently, the research community and, in particular, the statistical community have had major discussions on the appropriate use of $p$-values. Keeping this conversation going and refining how statistical reasoning can and should be done benefits quantitative research as a whole. Finally, statisticians can seek ways of extending open science to contexts with highly sensitive data. If data can be used without actually being seen by researchers (e.g., via distributed computing), results can be reproduced without breaching privacy. Statisticians are integral to making this possible.

\section{Roll up your sleeves!}

Publication bias, HARKing and many issues related to the use of null hypothesis significance testing in research are actually nothing new. Over 50 years ago, in 1966, the psychologist 
David Bakan wrote that his criticism concerning the use of statistical inference in psychological research was "hardly original" and that he was assuming "the role of the child who pointed out that the emperor was really outfitted only in his underwear". If many of the problems we are facing today have been known and criticised for many decades, yet remained unsolved, what are the chances that the scientific community will be able to change for the better during your 10-year hibernation?

Given the significant attention this topic has received in the last few years and the improvements that have been observed in some disciplines, we should not throw the towel in just yet. However, the involvement of statisticians in finding causes and solutions to the statistical crisis in science has been surprisingly limited and - with a few notable exceptions the main drivers have come from other disciplines. There is no doubt that we statisticians can contribute to improvements in research reproducibility and replicability across all applications of statistics. From our roles as data analysts, to the developers of tomorrow's methods: we are uniquely placed to debate the statistical crisis in science, develop solutions, and teach open replicable science. We have leverage potential to create change and we should use it. We must promote more efficient, replicable and credible science starting today by being the role model statisticians that we need for tomorrow.

\section{Acknowledgements}

We thank the DFG (individual grants BO3139/4-3 and BO3139/7-1) to ALB for funding, the German Federal Ministry of Education and Research (BMBF, Grant No. 01IS18036A) and the people on Twitter who helped improve our paper with their valuable comments and literature recommendations (see https://twitter.com/HeidiBaya/timelines/1158695192494559234).

\section{References}

Bakan, D. (1966). The test of significance in psychological research. Psychological Bulletin, 66(6), 423-437. doi:10.1037/h0020412

Botvinik-Nezer, R., Holzmeister, F., Camerer, C. F., Dreber, A., Huber, J., Johannesson, M., et al. (2020). Variability in the analysis of a single neuroimaging dataset by many teams. Nature, 582(7810), 84-88. doi:10.1038/s41586-020-2314-9

Boulesteix, A.-L., Hoffmann, S., Charlton, A. and Seibold, H. (2020), A replication crisis in methodological research?. Significance, 17: 18-21. doi:10.1111/1740-9713.01444

Colquhoun, D. (2014). An investigation of the false discovery rate and the misinterpretation of $p$-values. Royal Society Open Science, 1(3), 140216. doi:doi:10.1098/rsos.140216 
Daumer, M., Held, U., Ickstadt, K., Heinz, M., Schach, S., \& Ebers, G. (2008). Reducing the probability of false positive research findings by pre-publication validation - Experience with a large multiple sclerosis database. BMC Medical Research Methodology, 8(1), 18. doi:10.1186/1471-2288-8-18

Freedman, L. P., Cockburn, I. M., \& Simcoe, T. S. (2015). The Economics of Reproducibility in Preclinical Research. PLoS Biology, 13(6), e1002165. doi:10.1371/journal.pbio.1002165

Greenland, S., Senn, S. J., Rothman, K. J., Carlin, J. B., Poole, C., Goodman, S. N., et al. (2016). Statistical tests, P values, confidence intervals, and power: a guide to misinterpretations. European Journal of Epidemiology, 31(4), 337-350. doi:10.1007/s10654-016-0149-3

Hastings, J., Haug, K., \& Steinbeck, C. (2014). Ten recommendations for software engineering in research. GigaScience, 3(1). doi:10.1186/2047-217x-3-31

Held, L., \& Schwab, S. (2020). Improving the reproducibility of science. Significance, 17(1), 10-11.

doi:10.1111/j.1740-9713.2020.01351.x

Hoffmann, S., Schönbrodt, F. D., Elsas, R., Wilson, R., Strasser, U., \& Boulesteix, A. (2020, February 19). The multiplicity of analysis strategies jeopardizes replicability: lessons learned across disciplines.

https://doi.org/10.31222/osf.io/afb9p

Jacobsen, A., Azevedo, R. d. M., Juty, N., Batista, D., Coles, S., Cornet, R., et al. (2020). FAIR Principles:

Interpretations and Implementation Considerations. Data Intelligence, 2(1-2), 10-29. doi:10.1162/dint_r_00024

Manamley, N., Mallett, S., Sydes, M.R. et al. (2016). Data sharing and the evolving role of statisticians. BMC Med Res Methodol, 16, 75. doi:10.1186/s12874-016-0172-9

Nüst, D., Konkol, M., Pebesma, E., Kray, C., Schutzeichel, M., Przibytzin, H., et al. (2017). Opening the Publication Process with Executable Research Compendia. D-Lib Magazine, 23. doi:10.1045/january2017-nuest

Silberzahn, R., Uhlmann, E. L., Martin, D. P., Anselmi, P., Aust, F., Awtrey, E., et al. (2018). Many Analysts, One Data Set: Making Transparent How Variations in Analytic Choices Affect Results. Advances in Methods and Practices in Psychological Science, 1(3), 337-356. doi:10.1177/2515245917747646

Simmons, J. P., Nelson, L. D., \& Simonsohn, U. (2011). False-Positive Psychology:Undisclosed Flexibility in Data Collection and Analysis Allows Presenting Anything as Significant. Psychological Science, 22(11), 1359-1366.

doi:10.1177/0956797611417632

Trafimow, D., \& Marks, M. (2015). Editorial. Basic and Applied Social Psychology, 37(1), 1-2.

doi:10.1080/01973533.2015.1012991

Wasserstein, R. L., \& Lazar, N. A. (2016). The ASA Statement on p-Values: Context, Process, and Purpose. The American Statistician, 70(2), 129-133. doi:10.1080/00031305.2016.1154108

Wasserstein, R. L., Schirm, A. L., \& Lazar, N. A. (2019). Moving to a World Beyond " $p$ < 0.05 ". The American Statistician, 73(sup1), 1-19. doi:10.1080/00031305.2019.1583913 
The authors:

Heidi Seibold is the group lead of the working group Open Al in Health at Helmholtz Al, Helmholtz Zentrum München, Germany.

Alethea Charlton is a student assistant in the Institute for Medical Information Processing, Biometry and Epidemiology at LMU München, Germany.

Anne-Laure Boulesteix is a professor of biometrics in the Institute for Medical Information Processing, Biometry and Epidemiology at LMU München, Germany.

Sabine Homann is a postdoctoral researcher in the Institute for Medical Information Processing, Biometry and Epidemiology at LMU München, Germany 